Es war mir auch bei der dritten Auflage ein Vergnügen, mit MIT Press zusammenzuarbeiten. Ich danke Marie Lufkin Lee, Marc Lowenthal und Kathleen Caruso für ihre Hilfe und Unterstützung.

\title{
Hinweise zur zweiten englischen Auflage
}

Auf dem Gebiet des maschinellen Lernens gab es seit dem Erscheinen der ersten Auflage des Buches im Jahr 2004 bedeutende Entwicklungen. Erstens sind die Anwendungsgebiete enorm gewachsen. Internetbasierte Technologien wie Suchmaschinen, Empfehlungssysteme, Spamfilter und Angriffserkennungssysteme verwenden heute routinemäßig maschinelles Lernen. In der Bioinformatik werden in immer größerem Umfang Verfahren eingesetzt, die aus vorhandenen Daten lernen. Im Bereich der natürlichen Sprachverarbeitung - beispielsweise bei der maschinellen Übersetzung - beobachten wir eine sich beschleunigende Entwicklung weg von programmierten Expertensystemen und hin zu Verfahren, die automatisch aus einem sehr großen Korpus von Beispieltexten lernen können. Auch in der Robotik, in der medizinischen Diagnostik, bei der Sprachund Bilderkennung, in der Biometrie und im Finanzwesen gibt es immer mehr Anwendungen für die in diesem Buch vorgestellten Methoden des maschinellen Lernens, die auch unter Schlagwörter wie Mustererkennung, Data Mining und noch einigen anderen bekannt sind.

Zweitens gab es auch bei der Theorie hinter diesen Anwendungen große Fortschritte. So gestatten das Konzept der Kernel-Funktionen und die auf ihnen basierenden Kernel-Maschinen eine bessere Repräsentation des Problems. Die damit verbundene Methodik der komplexen Optimierung führt weiter als mehrlagige Perzeptronen mit sigmoidalen verborgenen Einheiten, die mittels Gradientenabstieg trainiert werden. Bayessche Methoden mit geeignet gewählten a-priori-Verteilungen fügen Expertenwissen zu dem hinzu, was uns die Daten verraten. Graphenmodelle erlauben die Darstellung durch ein Netzwerk von Knoten, und mit effizienten Inferenzalgorithmen sind Suchanfragen an solche Netzwerke möglich. Diese Entwicklungen erfordern es, die drei Themen - Kernel-Maschinen, Bayessche Schätzung und Graphenmodelle -, die in der ersten Auflage in Abschnitten behandelt wurden, ausführlicher zu betrachten, weshalb drei neue Kapitel hinzugekommen sind.

Von großer Bedeutung für das maschinelle Lernen war die Erkenntnis, dass Experimente auf der Basis maschinellen Lernens ein besseres Design brauchen. Aus diesem Grund habe ich in der zweiten Auflage das Kapitel über statistische Tests so umformuliert, dass es nun auch das Design und die Analyse von Experimenten mit maschinellem Lernen umfasst. Wichtig ist, dass das Testen nicht als separater Schritt aufgefasst werden sollte, der ausgeführt wird, wenn alle Durchläufe abgeschlossen sind (auch wenn das neue Kapitel am Ende des Buches steht); vielmehr sollte man den gesamten Prozess des Experimentierens vorab konzipieren, relevante 
Faktoren definieren, den richtigen Versuchsablauf wählen und erst dann die Durchläufe ausführen und die Daten analysieren.

Lange Zeit war die vorherrschende Meinung in der wissenschaftlichen Community, dass unser gegenwärtiges Wissen im Allgemeinen und das Wissen in der Computerwissenschaft im Besonderen nicht ausreicht, um Maschinen zu entwickeln, die so intelligent sind wie wir und dass künstliche Intelligenz aus diesem Grund nicht Realität werden könnte. Die meisten Leute glauben, dass wir eine neue Technologie brauchen, ein neues Material, neuartige Berechnungsmechanismen oder neuartige Programmiermethoden. Demnach könnten wir bis dahin nur einige Aspekte der menschlichen Intelligenz „simulieren“ und auch dies nur in eingeschränkter Form, weshalb sie letzlich unerreichbar bliebe.

Ich glaube, dass sich diese Ansicht schon bald als falsch erweisen wird. Zuerst haben wir dies beim Schach gesehen, und inzwischen beobachten wir Ähnliches in vielen Gebieten. Wenn wir nur genügend Speicher und Rechenleistung haben, können wir Aufgaben mithilfe relativ einfacher Algorithmen realisieren. Der Trick dabei ist das Lernen, entweder aus Beispieldaten oder durch bestärkendes Lernen nach dem Prinzip von Versuch und Irrtum. Es sieht danach aus, dass der Einsatz von Algorithmen für überwachtes und nahezu unüberwachtes Lernen - etwa im Bereich der maschinellen Übersetzung - schon bald möglich sein wird. Das Gleiche gilt für viele andere Gebiete, beispielsweise für die unbemannte Navigation in der Robotik mittels bestärkendem Lernen. Ich glaube, dass sich diese Entwicklung in vielen Bereichen der künstlichen Intelligenz fortsetzen wird, und der Schlüssel ist das Lernen. Wir müssen keine neuen Algorithmen einführen, wenn Maschinen selbst in der Lage sind zu lernen; wir müssen sie nur mit ausreichend großen Datenmengen füttern und genügend Rechenleistung zur Verfügung haben.

Ich möchte an dieser Stelle allen Lehrenden und Studierenden danken, die mit der ersten Auflage einschließlich dem Reprint in Indien und der deutschen Übersetzung gearbeitet haben. Besonders danke ich jenen, die mir Worte der Anerkennung und Errata zukommen ließen oder mir in anderer Weise Feedback gegeben haben. Bitte tun Sie das auch weiterhin. Meine E-Mail-Adresse ist alpaydin@boun.edu.tr.

Die zweite Auflage bietet auch im Internet in größerem Umfang Unterstützung unter http://www.cmpe.boun.edu.tr/ ethem/i2ml.

Danken möchte ich außerdem meinen ehemaligen und aktuellen Doktoranden Mehmet Gönen, Esma Kılıç, Murat Semerci, M. Aydın Ulaş und Olcay Taner Yıldız sowie den Hörern meiner Vorlesungen. Wenn man sein Wissen in einem bestimmten Fach testen möchte, ist der beste Weg der, dieses Fach zu unterrichten.

Es war mir auch bei der zweiten Auflage ein Vergnügen, mit MIT Press zu arbeiten. Ich danke Bob Prior, Ada Brunstein, Erin K. Shoudy, Kathleen Caruso und Marcy Ross für ihre Hilfe und Unterstützung. 